\title{
Efficient Singlet Oxygen Generation Upon Two-Photon Excitation of New Porphyrin With Enhanced Nonlinear Absorption
}

\author{
Aliaksandr Karotki, Mikalai Kruk, Mikhail Drobizhev, Aleksander Rebane, Eric Nickel, and Charles W. Spangler
}

\begin{abstract}
We demonstrate efficient generation of singlet oxygen upon two-photon excitation with 150 -fs 780 -nm laser pulses of a new porphyrin photosensitizer molecule whose two-photon absorption cross section has been considerably enhanced by chemical design.
\end{abstract}

Index Terms-Photodynamic therapy, porphyrin photosensitizers, singlet oxygen, two-photon absorption.

\section{INTRODUCTION}

A LONG-STANDING dream in the field of cancer therapy is the ability to treat subcutaneous tumors noninvasively, while at the same time eliminating the adverse physical discomfort associated with traditional chemotherapy and the debilitating effects of actinic radiation treatments. Photodynamic therapy (PDT) is gaining acceptance worldwide as an alternative treatment of tumors [1] as well as age-related macular degeneration [2]. PDT employs the special ability of some porphyrin photosensitizers to accumulate in pathologic cells and to transfer absorbed photon energy efficiently to extremely active singlet oxygen molecules, which then wipe out the surrounding tumor. Unfortunately, the limited penetration depth by visible light into biological tissue allows only few types of skin, breast, and certain endoscopically accessible cancers to be treated in this fashion. To make PDT more generally applicable, it is crucial to deliver light deeper into the tissue. This may be achieved by utilizing the nonlinear-optical effect of two-photon absorption (TPA) in which case the illumination is carried out at near-infrared (IR) wavelengths, where the tissue is significantly more transparent than in the visible. However, so far TPA of tumor-specific porphyrins has been notoriously inefficient, rendering the treatment of deeper tumors impractical. Here, we introduce a new porphyrin photosensitizer with an enhanced TPA cross

Manuscript received May 22, 2001. This work was supported by the Air Force Office of Scientific Research under Grant F49620-01-1-0324, Grant F49620-96-1-0440, Grant F49620-98-1-0422, and Grant F49620-98-1-0157.

A. Karotki and A. Rebane are with the Department of Physics, Montana State University, Bozeman, MT 59717 USA (e-mail: rebane@ physics.montana.edu).

M. Kruk was with the Department of Physics, Montana State University, Bozeman, MT 59717 USA. He is now with the Institute of Molecular and Atomic Physics, National Academy of Sciences, 220072 Minsk, Belarus.

M. Drobizhev is with the Department of Physics, Montana State University, Bozeman, MT 59717 USA and also with the P. N. Lebedev Physical Institute of the Russian Academy of Sciences, 117942 Moscow, Russia.

E. Nickel is with Synar Technologies, Inc., Atlanta, GA 30303 USA.

C. W. Spangler is with the Department of Chemistry and Biochemistry, Montana State University, Bozeman, MT 59717 USA.

Publisher Item Identifier S 1077-260X(01)11237-2.

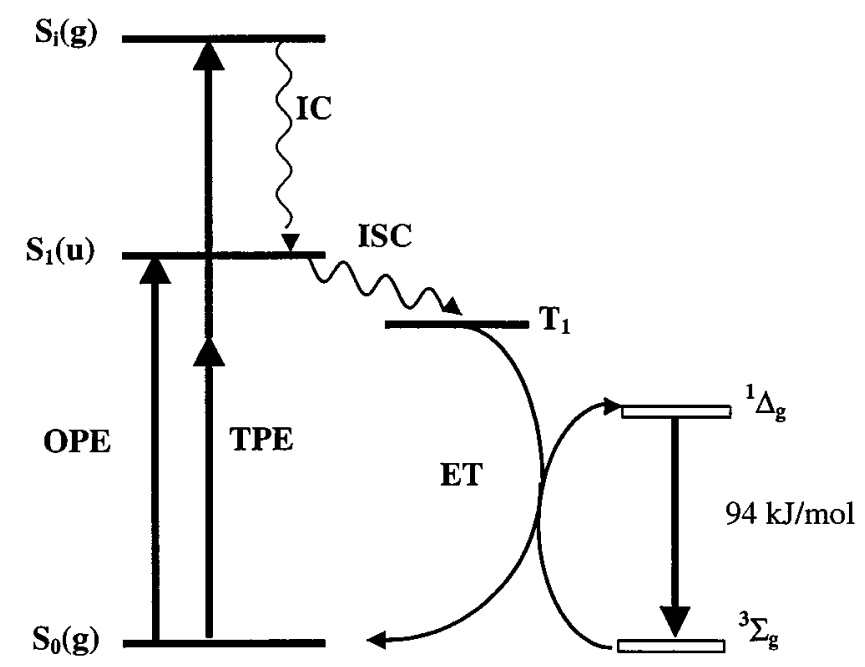

Fig. 1. Schematic of the energy levels for porphyrin photosensitizer (solid bars) and molecular oxygen (open bars). $S_{0}(\mathrm{~g}), S_{1}(\mathrm{u}), S_{i}(\mathrm{~g})$, and $T_{1}$ represent, respectively, ground, first singlet, $i$ th excited singlet, and lowest triplet states of the photosensitizer. The symbols in the parenthesises denote gerade $(\mathrm{g})$ and ungerade (u) symmetry of the corresponding states. ${ }^{3} \Sigma_{g}^{-}$and ${ }^{1} \Delta_{g}$ denote the ground and the first excited singlet states of molecular oxygen.

section, and demonstrate its ability to generate singlet oxygen upon illumination with near-IR light. Injecting a porphyrin photosensitizer into a patient's blood stream commences a typical PDT procedure. After an appropriate time interval (usually tens of hours), the photosensitizer is activated by shining a visible light, usually a red color laser beam, at the tumor's location [1], [3]. Photophysical processes constituting PDT are summarized in the energy level diagram shown in Fig. 1. In its classical implementation, absorption of one visible photon [one-photon excitation (OPE)] brings a photosensitizer molecule into a short-lived excited state $S_{1}$ with energy of 170-190 kJ.mol ${ }^{-1}$, which corresponds to an illumination wavelength of $\lambda=620-690 \mathrm{~nm}$. After a few nanoseconds, the porphyrin undergoes an intersystem crossing to a triplet state $T_{1}$ with energy of $110-130 \mathrm{~kJ} \cdot \mathrm{mol}^{-1}$ and with a much longer life time (on the order of milliseconds). From the triplet state, the energy is transferred (semicircle arrows in Fig. 1) to omnipresent oxygen molecules by switching them from a triplet ground state ${ }^{3} \Sigma_{g}^{-}$into an excited singlet state ${ }^{1} \Delta_{g}$ with excitation energy of $94 \mathrm{~kJ} \cdot \mathrm{mol}^{-1}$. Once in the excited singlet state, the oxygen presents an extremely reactive species capable of damaging surrounding tissue in an irreversible manner. A presence of excited singlet oxygen molecules $\left({ }^{1} \Delta_{g}\right)$ in solution is usually detected by their ${ }^{1} \Delta_{g} \rightarrow{ }^{3} \Sigma_{g}^{-}$luminescence at about $1270 \mathrm{~nm}$ (black arrow in Fig. 1). 
To be effective, the photosensitizers have to selectively accumulate in the tumor tissue. Serendipitously, porphyrin molecules possess this rare feature [3]. The second critical point is how deep can light penetrate into the body without being scattered or absorbed by normal tissue. It is well known that the tissue's transmission depends critically on the illumination wavelength [4] and is the largest in the so-called tissue transparency window at $\lambda \sim 750-1000 \mathrm{~nm}$. Porphyrins currently in use for PDT fall short of this transparency window: their $S_{1} \leftarrow S_{0}$ absorption varies from 620 to $690 \mathrm{~nm}$, where effective penetration in most tissues is no more than just a few millimeters in depth [5]. Unfortunately, attempts to shift the one-photon absorption band toward longer wavelengths by chemical modification of the porphyrin structure come into conflict with the fundamental requirement that the excitation energy of singlet oxygen is lower than the energy of the $T_{1}$ state. In addition, long-wavelength shift of porphyrin's energy levels often aggravates the situation by reducing compound's stability.

In the view of these mounting difficulties, a proposal to use TPA appears as the best alternative way to achieve PDT. TPA [6] is a third-order nonlinear-optical process and it consists in simultaneous absorption of two photons, so that the illumination wavelength is twice of that of the actual transition wavelength [two-photon excitation (TPE) in Fig. 1]. TPA allows using near-IR photons in the tissue transparency window and does not require the red shift of the lowest electronic transition of the porphyrin. Note that even if TPA takes a molecule into one of its' higher excited states $S_{i}$, this does not bar the lowest $S_{1}$ state to be populated via internal conversion. Once this state is formed, the subsequent events follow the same path as described above. In addition, TPA also provides two further advantages: 1) laser-induced hyperthermia is minimized, since near-IR light has a reduced absorption in tissues and 2) nonlinear (quadratic) dependence of TPA on laser intensity provides for higher spatial selectivity of treatment due to the smaller effective size of the focal spot. The latter is essential for treatment of sensitive tissues such as those of age-related wet macular degeneration. On the downside, because the probability of TPA with conventional low-power light sources is vanishing small, laser pulses with high peak intensity are required for the TPA effect to be pronounced. Fortunately, the commercialization of femtosecond mode-locked near-IR Ti : Sapphire lasers has greatly alleviated this last problem.

As one might expect, these useful features have stimulated an active search for new molecular system for TPA-based PDT [7]-[11]. Some nonporphyrin-based compounds may indeed possess an impressively large TPA cross section [8], [9], [12]. However, they either lack the vital property to generate singlet oxygen or their interaction with biological tissue is not known. On the other hand, all known porphyrin-based sensitizers were found to have a very small TPA cross section, typically not more than few Göppert-Mayer units (GM, $1 \mathrm{GM}=10^{-50} \mathrm{~cm}^{4}$ $\mathrm{s}_{\text {photon }}{ }^{-1}$ molecule $^{-1}$ ) [10] and, thus, require unreasonable prolonged illumination with a tightly focused laser beam [10], [11]. Our approach lies in special chemical modification of

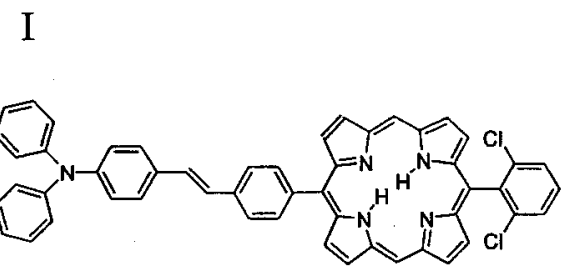

(a)

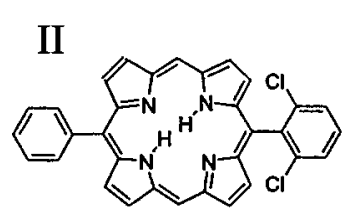

(b)

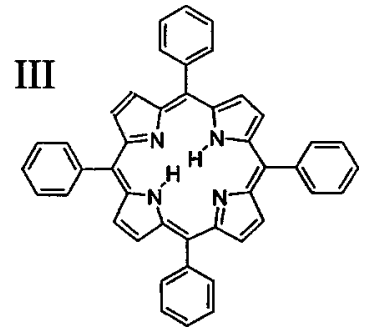

(c)

Fig. 2. Molecular structures of porphyrins studied. (a) 5-(4-diphenylaminostilbene), 15-(2, 6-dichlorophenyl)-21H, 23H-porphine. (b) 5-phenyl, 15-(2, 6-dichlorophenyl)-21H, 23H-porphine. (c) 5, 10, 15, 20-tetraphenyl-21H, 23H-porphine.

porphyrin, which enhances the molecule's TPA cross section without jeopardizing its ability to generate singlet oxygen. The design was based on structure-property relationships, known to enhance TPA cross section in organic $\pi$-conjugated chromophores [12]-[14]. In particular, introduction of donors or acceptors of electronic density as substituent groups, extension of $\pi$ conjugation, and inclusion of donor- $\pi$-donor motifs within chromophore have all been previously utilized. In our particular case, this was achieved by an introduction of a 4-(diphenylaminostilbene)-substituent into the mesoposition of the tetrapyrrol ring. This results in a 20 times enhancement in TPA cross section at $\lambda_{\text {exc }}=780 \mathrm{~nm}$. In addition, the inclusion of the 2, 6-dichlorophenyl substituent assists intersystem crossing via an internal heavy atom effect.

\section{EXPERIMENTAL SECTION}

Structures of a typical new porphyrin DPASP [5-(4-diphenylaminostilbene), 15-(2, 6-dichlorophenyl)-21H, 23H-porphine] (I), as well as its nonsubstituted counterpart (II) and commercial tetraphenylporphyrin (III) are shown in Fig. 2. The last two porphyrins were studied for purpose of comparison as model compounds. Compound I has been synthesized by condensing 10-(2, 6-dichlorophenyl) bilane with (4-diphenylaminostilbene) aldehyde under acidic conditions followed by oxidation with 2, 3-dichloro-5, 6-cyano-1, 4-benzoquinone. The way of synthesis of compound II is identical to that of I. Porphyrins I and II were purified by flash chromatography on silica using 20\%-40\% dichloromethane/hexane gradient and the structure of molecules was confirmed by spectroscopic analysis. Compound III was purchased from Aldrich and used as received.

Our laser system is schematically shown in Fig. 3. It comprised a Ti : sapphire regenerative amplifier (CPA-1000, Clark MRX), which was operated at $1-\mathrm{kHz}$ repetition rate and produced 150 -fs pulses at $0.8 \mathrm{~mJ}$ energy per pulse. These pulses 


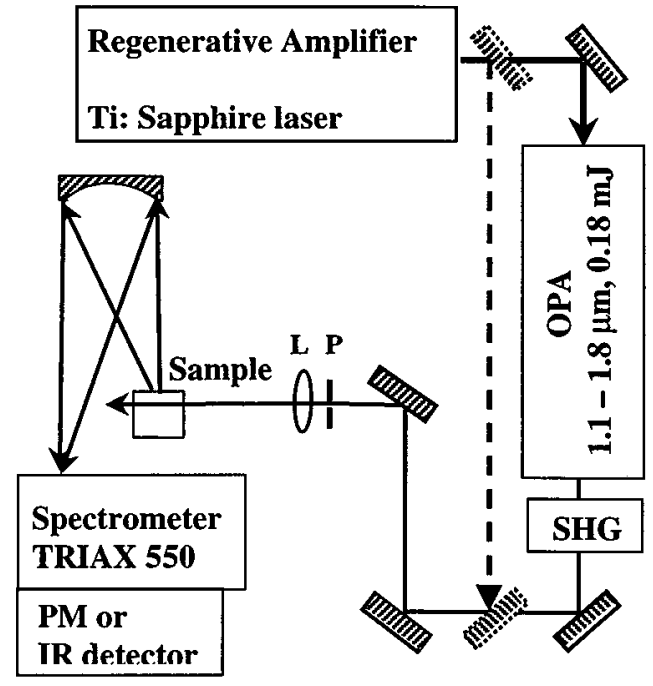

Fig. 3. Schematic of experimental setup. For measurement of TPA spectra, a second harmonic of tunable OPA was used. For measurements of TPA cross sections and singlet oxygen two-photon photo generation, the output of regenerative amplifier was used directly (dashed arrow). For one-photon singlet oxygen photosensitization, the second harmonic of this radiation was used.

were parametrically down-converted in the optical parametric amplifier (OPA) (TOPAS, Quantronix), which yielded 100-fs pulses in the range from 1.1 to $1.8 \mu \mathrm{m}$. TPA spectra were obtained by tuning the OPA with subsequent second-harmonic generation and registration of the porphyrin fluorescence. $\mathrm{Ab}$ solute TPA cross sections were measured by comparing fluorescence intensity under OPE and TPE (see [15] for details). For sech ${ }^{2}$ temporal profile of excitation pulses and under the assumption that fluorescence quantum efficiency is the same for both modes of excitation, the two-photon cross section writes as follows:

$$
\sigma_{2}=\frac{F_{2}}{F_{1}} \frac{\tau r\left(1-10^{-O D_{1}}\right)}{0.3 C l} \frac{I_{1}}{I_{2}^{2}} \frac{\left(h \nu_{2}\right)^{2}}{h \nu_{1}} \frac{t_{1}}{t_{2}}
$$

Here, indexes 1 and 2 refer to the values measured under OPE and TPE, respectively, $F$ is the fluorescence signal recorded during time $t, C$ is the molecule concentration (in molecule $\mathrm{cm}^{-3}$ ), $l$ is the sample thickness (in $\mathrm{cm}$ ), $\tau$ is the pulse duration (full width at half maximum in $\mathrm{s}$ ), $r$ is the pulse repetition rate under TPE, $I$ is the average intensity (in $\mathrm{W} / \mathrm{cm}^{2}$ ), $\nu$ is laser frequency (in hertz), and $O D_{1}$ is the optical density of the sample at one-photon illumination wavelength.

In our experiments, we employed the fundamental of Ti-sapphire regenerative amplifier for TPE and its second harmonic for corresponding OPE. In both cases, the beam was slightly focused with a $f=500 \mathrm{~mm}$ lens (L) into the $1-\mathrm{cm}$ cell with sample solution through a pinhole $(\mathrm{P})$ placed in front of the lens. Fluorescence was collected and focused on the entrance slit of a Jobin-Yvon monochromator with a spherical mirror. Special care were taken to geometrically eliminate the possible reabsorption effects.

In experiments with singlet oxygen generation, OPE was carried out at $\lambda=390 \mathrm{~nm}$ with average intensity of $0.5 \mathrm{~W} / \mathrm{cm}^{2}$ and TPE was carried out at $\lambda=780 \mathrm{~nm}$ with average intensity of $15 \mathrm{~W} / \mathrm{cm}^{2}$. The laser beam was slightly focused to give a cylin- drical irradiated volume of $\sim 1.5$-mm diameter in both cases. The singlet oxygen luminescence spectrum was measured with a nitrogen-cooled Ge detector coupled with monochromator and lockin amplifier.

\section{RESULTS AND DISCUSSION}

TPA spectra of the three molecules are shown in Fig. 4. Black solid lines represent the appropriately scaled one-photon absorption. All three molecules reveal a characteristic strong Soret band near $415 \mathrm{~nm}$, accompanied by four relatively weak bands in the visible (not shown).

We observe that the TPA band does not coincide with any of the bands in the linear absorption spectrum. Quantum-mechanical calculations [16], [17] show that key spectroscopic properties of porphyrins such as position and symmetry of main electronic energy levels are determined by the tetrapyrrol macrocycle and predict that the lowest excited state of $B_{g}$ symmetry should be positioned near the Soret band. According to selection rules, centrosymmetric molecules should have allowed onephoton transitions between the states of opposite parity and twophoton transitions between the states of same parity. Since the porphin ground state has $A_{g}$ symmetry, we assume that the observed TPA bands correspond to the transitions between the two states of $g$ parity.

The key point, however, is that DPASP (I) shows a very large (for porphyrins) TPA cross section with a measured maximum value of $80 \pm 10 \mathrm{GM}$ at about $390 \mathrm{~nm}\left(\lambda_{\text {exc }}=780 \mathrm{~nm}\right)$. The most significant fact is that this excitation wavelength falls well into the tissue transparency window, which makes the new approach very promising for future applications of PDT.

Fig. 5 demonstrates that we were able to directly detect the production of singlet oxygen upon TPA of DPASP (I). Fig. 5(a) shows two luminescence spectra obtained upon either one-photon (dash) or two-photon (solid) excitation of DPASP, both normalized to unity. Note that the two spectra coincide well within experimental error and show both a characteristic peak near $1276 \mathrm{~nm}$, which undoubtedly corresponds to the singlet oxygen luminescence spectrum [18]. Fig. 5(b) shows that the intensity of luminescence upon TPE increases as a square of the illumination intensity, which proves that the porphyrin is indeed excited by absorbing two near-IR photons simultaneously. Furthermore, if the solution was treated with a nitrogen sparge, the intensity of luminescence decreased because of the decreased oxygen concentration. Note that only with DPASP (I) could we reliably detect oxygen luminescence upon TPA. In the case of II and III, while a strong luminescence could be obtained upon OPE, with infrared excitation the signal was below the noise level of our detector.

\section{CONCLUSION}

We have demonstrated, for the first time to the best of our knowledge, that singlet oxygen can be produced efficiently upon TPE of porphyrin photosensitizer with near-IR illumination within the tissue transparency window. This is achieved by chemically attaching special moieties to the porphyrin molecule, which are increasing the TPA cross section in near-IR. We believe that our results open new possibilities for designing 


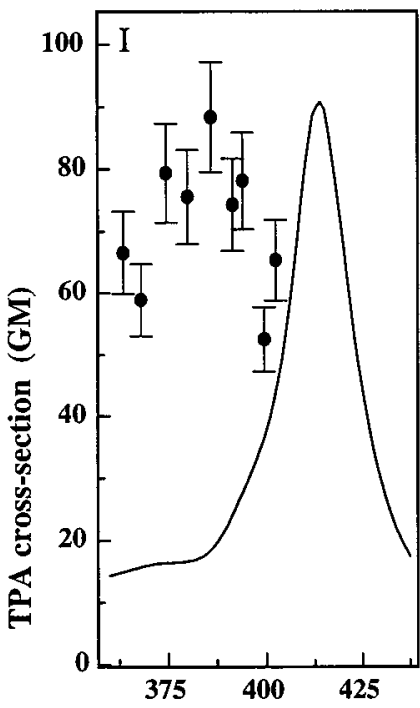

(a)

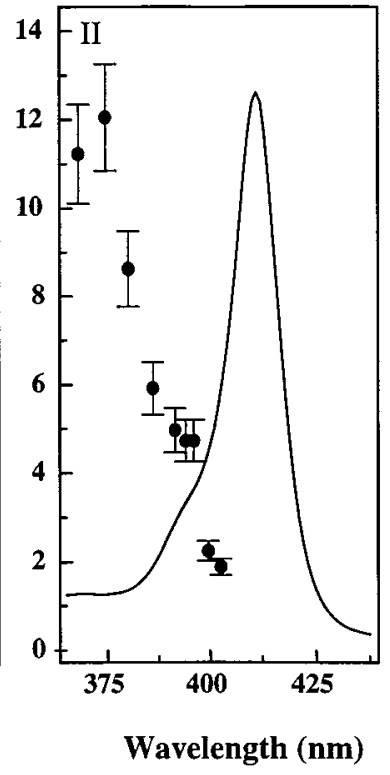

(b)

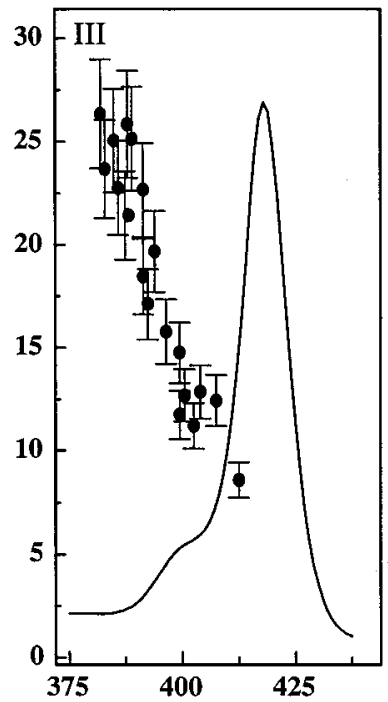

(c)

Fig. 4. TPA spectra (circles) of $10^{-4} \mathrm{M}$ toluene solutions of compounds (a) I, (b) II, and (c) III. The spectra could not be measured at wavelength shorter than shown because the energy of excitation photon approaches that of the first excited singlet state $S^{1}$ and efficient one-photon absorption masks TPA. The wavelength axis corresponds to the transition wavelength, i.e., half the laser wavelength. Vertical axis shows the TPA cross sections in Göppert-Mayer.
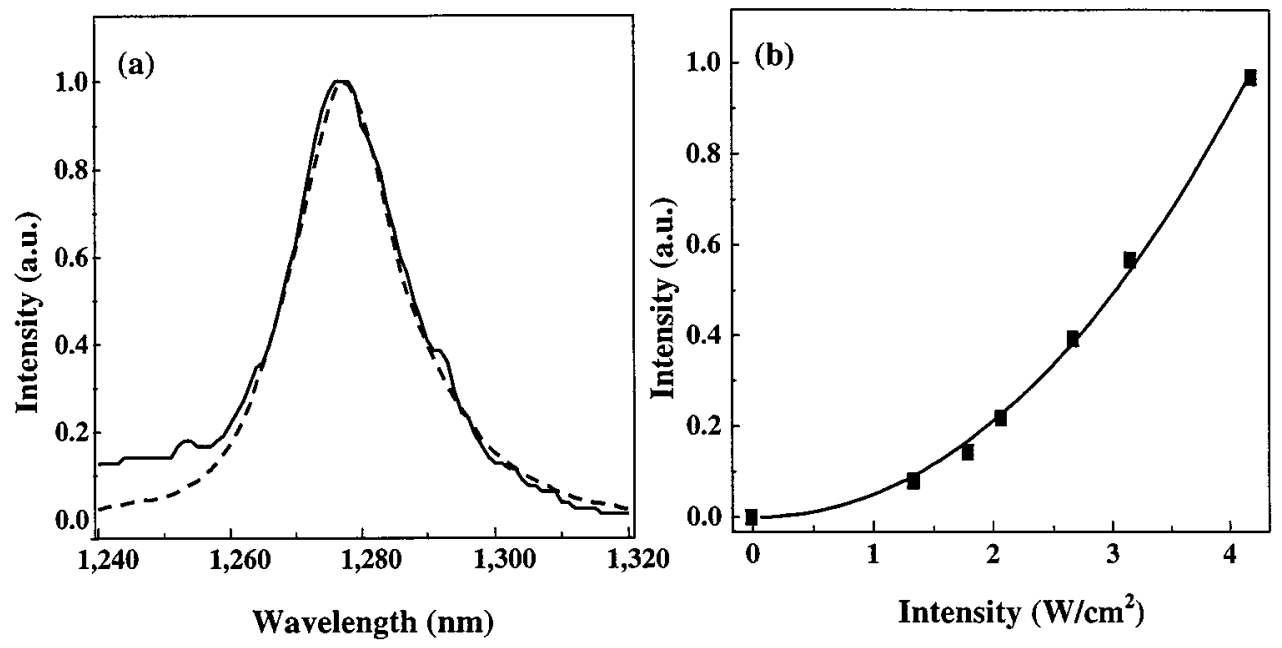

Fig. 5. (a) ${ }^{1} \Delta_{g} \rightarrow{ }^{3} \Sigma_{g}^{-}$luminescence spectra of molecular oxygen in air-saturated toluene solution of I. Dashed and solid curves represent, respectively, the spectra measured with OPE and TPE. Both spectra are normalized to unity. (b) Dependence of the ${ }^{1} \Delta_{g} \rightarrow{ }^{3} \Sigma_{g}^{-}$oxygen luminescence intensity $I_{\Delta}$ on the average illumination intensity, $P$, upon TPE of porphyrin. Experimental data are shown by black squares. Solid curve is the best power-law fit $I_{\Delta}=a P^{n}$ with $n=2.1 \pm 0.1$.

tumor-specific drugs with a large TPA cross section, which may eventually lead to PDT-based treatment of various deep tumors.

\section{ACKNOWLEDGMENT}

The authors would like to thank R. Cone for his generous loan of a Ge-detector.

\section{REFERENCES}

[1] B. W. Henderson and T. J. Dougherty, "How does photodynamic therapy work?," Photochem. Photobio., vol. 55, no. 1, pp. 147-157, Jan. 1992.

[2] N. Bressler et al., "Photodynamic therapy of subfoveal choroidal neovascularization in age-related macular degeneration with verteporfin. One-year results of 2 randomized clinical trials-TAP Report 1," Arch. Ophthalmol., vol. 117, no. 2, pp. 1329-1348, Oct. 1999.
[3] R. Bonnett, "Photosensitizers of the porphyrin and phtalocyanine series for photodynamic therapy," Chem. Soc. Rev., vol. 24, no. 1, pp. 19-33, Feb. 1995.

[4] S. Wan, J. A. Parrish, R. R. Anderson, and M. Madden, "Transmittance of nonionizing radiation in human tissues," Photochem. Photobio., vol. 34, pp. 679-681, 1981.

[5] W.-F. Cheong, S. A. Prahl, and A. J Welch, "A review of the optical properties of biological tissues," IEEE J. Quantum Electron., vol. 26, pp. 2166-2185, Dec. 1990.

[6] S. Kershaw, "Two-photon absorption," in Characterization Techniques and Tabulations for Organic Nonlinear Optical Materials, M. G. Kuzyk and C. W. Dirk, Eds. New York: Marcel Dekker, 1998.

[7] W. G. Fisher, W. R. Partridge, Jr., C. Dees, and E. A. Wachter, "Simultaneous two-photon activation of type-I photodynamic therapy agents," Photochem. Photobio., vol. 66, no. 2, pp. 1415-155, Aug. 1997.

[8] J. D. Bhawalkar, N. D. Kumar, C.-F. Zhao, and P. N. Prasad, "Twophoton photodynamic therapy," J. Clin. Lasers Med. Surg., vol. 15, no. 5, pp. 201-204, Sept.-Oct. 1997. 
[9] P. K. Frederiksen and O. P. R. Jørgensen, “Two-photon photosensitized production of singlet oxygen," J. Amer. Chem. Soc., vol. 123, no. 6, pp. 1215-1221, Feb. 2001.

[10] R. L. Goyan and D. T. Cramb, "Near-infrared two-photon excitation of protoporphyrin IX: Photodynamics and photoproduct generation," Photochem. Photobio., vol. 72, no. 6, pp. 821-827, Dec. 2000.

[11] K. König, "Multiphoton microscopy in life sciences," J. Microsc., pt. 2, vol. 200, pp. 83-104, Nov. 2000

[12] M. Albota et al., "Design of organic molecules with large two-photon absorption cross sections," Science, vol. 281, no. 5383, pp. 1653-1656, Sept. 1998.

[13] B. H. Cumpston et al., "Two-photon polymerization initiators for three-dimensional optical data storage and microfabrication," Nature, vol. 398, no. 6722, pp. 51-54, Mar. 1999

[14] C. W. Spangler, "Recent developments in the design of organic materials for optical power limiting," J. Mater. Chem., vol. 9, no. 9, pp. 2013-2020, Sept. 1999.

[15] M. Drobizhev, A. Karotki, and A. Rebane, "Persistent spectral hole burning by simultaneous two-photon absorption," Chem. Phys. Lett., vol. 334, no. 1-3, pp. 76-82, Feb. 2001.

[16] V. A. Kuz'mitsky and K. N. Solov'ev, "PPDP/S calculation of the porphin molecule electronic spectra," J. Appl. Spectr., vol. 27, pp. 724-730, 1977.

[17] D. Sundholm, "Density functional theory study of the electronic absorption spectrum of Mg-porphyrin and Mg-etioporphyrin-I," Chem. Phys. Lett., vol. 317, no. 3-5, pp. 392-399, Feb. 2000.

[18] A. Bromberg and C. S. Foote, "Solvent shift of singlet oxygen emission wavelength," J. Phys. Chem., vol. 93, no. 10, pp. 3968-3969, May 1989.

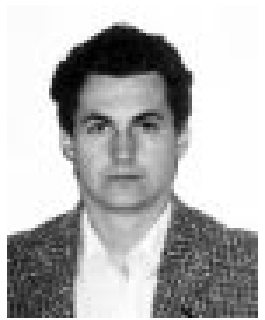

Aliaksandr Karotki was born in Minsk, Belarus, in 1978. He received the M.S. degree in physics with a specialization in laser physics and spectroscopy from the Belarus State University, Minsk, Belarus, in 1998. $\mathrm{He}$ is currently working toward the Ph.D. degree at Montana State University, Bozeman.

He was with the Institute of Molecular and Atomic Physics of the Belarus National Academy of Science, Minsk, Belarus. His current research interests include nonlinear optics of organic materials and their applications.

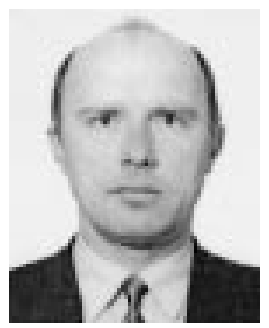

Mikalai Kruk was born near Minsk, Belarus, in 1967. He received the M.S. degree in physics from the Belarussian State University, Minsk, Belarus, in 1991 and the Ph.D. degree in physics from the Institute of Molecular and Atomic Physics of the National Academy of Science of Belarus, Minsk, Belarus, in 1997.

He was in the Soviet Army and was with the University of P. and M. Curie from 1997 to 1998 . He has been with the National Academy of Sciences of Belarus since 1998. He had a short visit with the Montana State University, Bozeman, in 2001. He has authored or coauthored over 30 papers. His current research interests include the photophysics and photochemistry of porphyrins and related compounds.

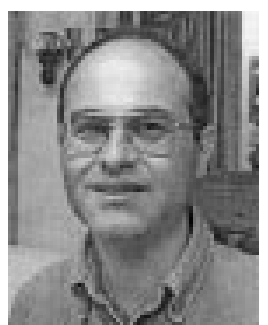

Mikhail Drobizhev was born in Moscow, Russia, in 1963. He received the M.S. degree in physics with a specialization in molecular biophysics from the Moscow Institute of Physics and Technology, Moscow, Rusia, and Ph.D. degree in physics from the P. N. Lebedev Physical Institute of the Russian Academy of Sciences, Moscow, Russia, in 1998.

Since 1986, he has been an Associate Researcher with the P. N. Lebedev Physical Institute of the Russian Academy of Sciences and was a Research Scientist under Prof. Rebane with Montana State University, Bozeman, in 1999. He has authored or coauthored over 40 papers. His current research interests include selective laser spectroscopy of porphyrins and other biological molecules and their applications in data storage, optoelectronics, and laser medicine.

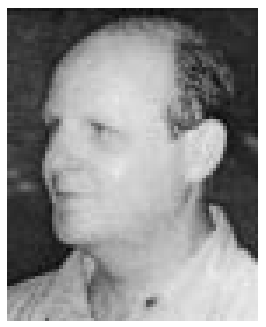

Aleksander Rebane received the M.S. degree in physics from the Tartu University, Tartu, Estonia, in 1981 and the Ph.D. degree in physics from the Estonian Academy of Sciences, Tartu, Estonia, in 1985.

From 1991 to 1997, he was Oberassistent and Privatdozent with the Institute for Physical Chemistry, the Swiss Federal Institute of Technology (ETH), Zurich, Switzerland. He received from the ETH Habiliation in physical chemistry in 1996 and became an Associate Professor of Physics with Montana State University, Bozeman, in 1997. His current research interests include nonlinear optics of organic materials, ultrafast optical storage, time-and-space-domain holography, ultrafast spectroscopy of organic molecules, and medical applications of nonlinear optics.

Dr. Rebane received the Intenational Commission for Optics Prize in 1993 , the Leopold Ruzicka Prize in 1996, and the Charles and Nora Wiley Award in 1999.

Eric Nickel, photograph and biography not available at the time of publication.

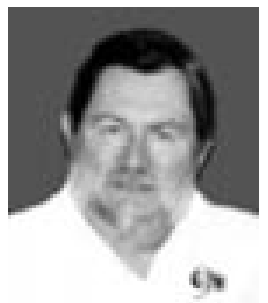

Charles W. Spangler reeived the B.S. degree in chemistry from the Massachusetts Institute of Technology, Cambridge, in 1959, the M.S. degree in organic chemistry from Northeastern University, Boston, MA, in 1961, and the Ph.D. degree from the University of Maryland, College Park, in 1964.

After on year as a Kettering Foundation TeacherIntern with Ohio Wesleyan University, he joined the Faculty of Northern Illinois University as an Assistant Professor or Organic Chemistry in 1965, where he became an Associate Professor in 1972, Professor in 1981, a Presidential Research Professor in 1990, and a Distinguished Research Professor in 1994. In 1996, he became a Research Professor with the Department of Chemistry and Biochemistry and the Optical Center, Montana State University, Bozeman. He has authored or coauthored more than 180 research papers and has presented at more than 125 national and international meetings and symposia. 